\title{
Quadratic symmetry of modified $q$-Euler polynomials
}

\author{
SangKi Choi ${ }^{1}$, Taekyun Kim², Hyuck-In Kwon² and Jongkyum Kwon ${ }^{3 *}$
}

\section{"Correspondence:}

mathkjk26@gnu.ac.kr

${ }^{3}$ Department of Mathematics

Education and ERI, Gyeongsang

National University, Jinju, Republic of Korea

Full list of author information is

available at the end of the article

\begin{abstract}
We use the $p$-adic $q$-integral and group action to count the number of the generating functions of modified $q$-Euler polynomials in a prescribed set. Some generating function yields modified $q$-Euler polynomials with the isotropy group $D_{4}$ and some gives Euler polynomials with the isotropy group $V_{4}$.
\end{abstract}

MSC: $11 \mathrm{~B} 83 ; 42 \mathrm{~A} 16$

Keywords: $p$-adic $q$-integral; modified $q$-Euler polynomials; group action

\section{Introduction}

We attribute a substantial study of Bernoulli polynomials and Euler polynomials to Carlitz. He used an inductive method to define these special polynomials (see [1]).

In this paper we use the $p$-adic $q$-integral to define the special polynomials. It is Kim who has established the theory of the $p$-adic $q$-integral (see [2]). The $p$-adic $q$-integral provides fruitful and essential results in studying special polynomials such as Bernoulli, Euler and Catalan polynomials. One of such results is the symmetry of the special polynomials.

Throughout this paper $p$ is a fixed odd prime number. We use the notations $\mathbb{Z}_{p}, \mathbb{Q}_{p}$ and $\mathbb{C}_{p}$ to express the ring of $p$-adic integers, the field of $p$-adic rational numbers and the completion of algebraic closure of $\mathbb{Q}_{p}$, respectively. The $p$-adic norm $|\cdot|_{p}$ is normalized as $|p|_{p}=\frac{1}{p}$. For $q, x \in \mathbb{C}_{p}$ with $|q-1|_{p}<p^{-\frac{1}{p-1}}$. We define the $q$-analogue of a number $x$ to be $[x]_{q}=\frac{1-q^{x}}{1-q}$. Note that $\lim _{q \rightarrow 1}[x]_{q}=x$.

Let $U D\left(\mathbb{Z}_{p}\right)$ be the space of uniformly differentiable functions on $\mathbb{Z}_{p}$ and $f \in U D\left(\mathbb{Z}_{p}\right)$. Kim has introduced the fermionic $p$-adic $q$-integral $I_{-q}(f)$ on $\mathbb{Z}_{p}$ (see $[1,3-10]$ ).

$$
I_{-q}(f)=\int_{\mathbb{Z}_{p}} f(x) d u_{-q}(x)=\lim _{N \rightarrow \infty} \frac{1}{\left[p^{N}\right]_{-q}} \sum_{x=0}^{p^{N}-1} f(x)(-q)^{x} .
$$

The $q$-Euler polynomials $\mathcal{E}_{n, q}(x)$ are defined by the generating function

$$
\int_{\mathbb{Z}_{p}} e^{[x+y]_{q} t} d \mu_{-q}(y)=\sum_{n=0}^{\infty} \mathcal{E}_{n, q}(x) \frac{t^{n}}{n !} \quad(\text { see }[2,10-18]) .
$$

(0) The Author(s) 2018. This article is distributed under the terms of the Creative Commons Attribution 4.0 International License (http://creativecommons.org/licenses/by/4.0/), which permits unrestricted use, distribution, and reproduction in any medium, provided you give appropriate credit to the original author(s) and the source, provide a link to the Creative Commons license, and indicate if changes were made. 
Also the modified $q$-Euler polynomials $E_{n, q}(x)$ are defined by the generating function

$$
\int_{\mathbb{Z}_{p}} e^{[x+y]_{q} t} d \mu_{-1}(y)=\sum_{n=0}^{\infty} E_{n, q}(x) \frac{t^{n}}{n !} \quad(\text { see }[19,20])
$$

Note that $\mathcal{E}_{n, q}(x)=\int_{\mathbb{Z}_{p}}[x+y]_{q}^{n} d \mu_{-q}(y)$ and $E_{n, q}(x)=\int_{\mathbb{Z}_{p}}[x+y]_{q}^{n} d \mu_{-1}(y)$.

For $v$ and $w$ odd positive integers, put $p(x, u, v, w)=\sum_{i=0}^{v-1}(-1)^{i} \int_{\mathbb{Z}_{p}} e^{[u x+v y+w i]_{q} t} d \mu_{-1}(y)$. Then

$$
\begin{aligned}
p(x, u, v, w) & =\sum_{i=0}^{v-1}(-1)^{i} \int_{\mathbb{Z}_{p}} e^{[u x+v y+w i]_{q} t} d \mu_{-1}(y) \\
& =\sum_{i=0}^{v-1}(-1)^{i} \lim _{N \rightarrow \infty} \sum_{y=0}^{p^{N}-1} e^{[u x+v y+w i]_{q} t}(-1)^{y} \\
& =\sum_{i=0}^{v-1}(-1)^{i} \lim _{N \rightarrow \infty} \sum_{y=0}^{p^{N}-1} \sum_{k=0}^{w-1} e^{[u x+v(k+w y)+w i]_{q} t}(-1)^{k+w y} \\
& =\sum_{i=0}^{v-1} \sum_{k=0}^{w-1}(-1)^{i+k} \lim _{N \rightarrow \infty} \sum_{y=0}^{p^{N}-1} e^{[u x+v w y+v k+w i]_{q} t}(-1)^{y} .
\end{aligned}
$$

Note that the last line is invariant under the transposition $(v, w)$. So we obtain the 'basic' symmetry of the generating functions

$$
p(x, u, v, w)=p(x, u, w, v)
$$

In this paper we use four weights $w_{1}, w_{2}, w_{3}, w_{4}$ instead of $u, v, w$. Then the symmetric group $S_{4}$ of degree 4 naturally acts on the generating functions. We shall describe the group action more generally. Let $p=p\left(x, w_{1}, \ldots, w_{n}\right)$ be generating functions with a variable $x$ and $n$ weights $w_{1}, \ldots, w_{n}$ involved in the definition of $p$. For $\sigma \in S_{n}$, define

$$
\sigma\left(p\left(x, w_{1}, \ldots, w_{n}\right)\right)=p\left(x, w_{\sigma}(1), \ldots, w_{\sigma}(n)\right)
$$

Then it is obvious that (i) $i d(p)=p$ and (ii) $(\sigma \tau)(p)=(\sigma(\tau(p)))$ hold. Hence this is a group action naturally.

In this paper we will consider a set $X$ of 315 generating functions, $p=p\left(x, w_{1}, \ldots, w_{4}\right)$, and let $S_{4}$ act on this set. There will be 19 orbits in $X$ under the action of $S_{4}$, and 4 nonisomorphic subgroups of $S_{4}$ as the isotropy groups.

Throughout this paper $w_{1}, w_{2}, w_{3}, w_{4}$ are odd positive integers. Consider the generating functions $p(x)$, where

$$
p(x)=\sum_{i=0}^{w_{j} w_{k}-1}(-1)^{i} \int_{\mathbb{Z}_{p}} e^{\left[\left(w_{a} w_{b}+w_{c} w_{d}\right) x+w_{j} w_{k} y+w_{l} w_{m} i\right]_{q} t} d \mu_{-1}(y),
$$

where $1 \leq a, b, c, d, j, k, l, m \leq 4, a \neq b, c \neq d, j \neq k, l \neq m$ and $w_{a} w_{b} \neq w_{c} w_{d}$. That is, we use square-free quadratic forms of $w^{\prime} s$. 
By the abuse of notation, we denote $p(x)$ by $p(a b+c d, j k, l m)$. That is,

$$
p(a b+c d, j k, l m)=\sum_{i=0}^{w_{j} w_{k}-1}(-1)^{i} \int_{\mathbb{Z}_{p}} e^{\left[\left(w_{a} w_{b}+w_{c} w_{d}\right) x+w_{j} w_{k} y+w_{l} w_{m} i\right]_{q} t} d \mu_{-1}(y) .
$$

Then, due to the 'basic' symmetry in (1.5), $p(a b+c d, j k, l m)=p(a b+c d, l m, j k)$. Let $X$ be the set of all $p(a b+c d, j k, l m)$. Then there are $15 \times 6$ generating functions of the form $p(a b+c d, j k, j k)$ and $15 \times 15$ generating functions of the form $p(a b+c d, j k, l m)$, where $w_{j} w_{k} \neq w_{l} w_{m}$. So $X$ is a set of 315 generating functions.

Let the symmetric group $S_{4}$ act on $X$ naturally. That is, for any $\sigma \in S_{4}$,

$$
\sigma(p(a b+c d, j k, l m))=p(\sigma(a) \sigma(b)+\sigma(c) \sigma(d), \sigma(j) \sigma(k), \sigma(l) \sigma(m))
$$

We will find each orbit and the isotropy subgroup of $S_{4}$ of an element in the orbit. Note that we have the basic counting of group action such that the product of the cardinality of an orbit and that of the corresponding isotropy subgroup is $\left|S_{4}\right|=24$.

\section{Quadratic symmetry of the modified $q$-Euler polynomials with the isotropy group $D_{4}$}

We use the notation in (1.8) and put $f_{1}=p(12+34,12,34), f_{2}=p(13+24,13,24)$ and $f_{3}=$ $p(14+24,14,23)$. Then, for $\tau=(2,3,4)$ and $\tau^{2}=(2,4,3)$,

$$
\begin{aligned}
\begin{aligned}
\tau\left(f_{1}\right) & =\tau(p(12+34,12,34))=p(\tau(1) \tau(2)+\tau(3) \tau(4), \tau(1) \tau(2), \tau(3) \tau(4)) \\
& =p(13+42,13,42)=p(13+24,13,24)=f_{2},
\end{aligned} \\
\tau^{2}\left(f_{1}\right)=\tau^{2}(p(12+34,12,34))=p(14+23,14,23)=f_{3} .
\end{aligned}
$$

Hence $f_{1}, f_{2}, f_{3}$ are in an orbit under the action of $S_{4}$ on $X$.

Now consider a 4-cycle $\sigma_{1}=(1,3,2,4)$ and a 2 -cycle $\sigma_{2}=(1,2)$. It is obvious that $\sigma_{2}$ fixes $f_{1}$. Note that $w_{1} w_{2}+w_{3} w_{4}=w_{3} w_{4}+w_{1} w_{2}$. By the basic symmetry in (1.5), we obtain

$$
\begin{aligned}
\sigma_{1}(p(12+34,12,34)) & =p\left(\sigma_{1}(1) \sigma_{1}(2)+\sigma_{1}(3) \sigma_{1}(4), \sigma_{1}(1) \sigma_{1}(2), \sigma_{1}(3) \sigma_{1}(4)\right) \\
& =p(34+12,34,12)=p(12+34,12,34)
\end{aligned}
$$

That is, $\sigma_{1}$ fixes $f_{1}$ also. Hence the dihedral group $D_{4}=\langle(1,3,2,4),(1,2)\rangle$ is a subgroup of the isotropy subgroup of $f_{1}$. However, we find three elements $f_{1}, f_{2}, f_{3}$ in the orbit of $f_{1}$ and $\left|D_{4}\right|=8$. Thus $X_{1}=\left\{f_{1}, f_{2}, f_{3}\right\}$ is the orbit of $f_{1}$ and $D_{4}=\langle(1,3,2,4),(1,2)\rangle$ is the isotropy subgroup of $S_{4}$ fixing $f_{1}$.

Further, we can check that the cyclic group $\langle(2,3,4)\rangle$ acts transitively on $X_{1}$. That is,

$$
\begin{aligned}
& (2,3,4)\left(f_{1}\right)=(2,3,4)(p(12+34,12,34))=p(13+42,13,42)=f_{2}, \\
& (2,4,3)\left(f_{1}\right)=(2,4,3)(p(12+34,12,34))=p(14+23,14,23)=f_{3} .
\end{aligned}
$$


Theorem 2.1 Let $X$ be the set of all generating functions of the form

$$
p(a b+c d, j k, l m)=\sum_{i=0}^{w_{j} w_{k}-1}(-1)^{i} \int_{\mathbb{Z}_{p}} e^{\left[\left(w_{a} w_{b}+w_{c} w_{d}\right) x+w_{j} w_{k} y+w_{l} w_{m} i\right]_{q} t} d \mu_{-1}(y)
$$

and $S_{4}$ act on $X$ by permuting $w_{1}, w_{2}, w_{3}, w_{4}$. Take $f_{1}=p(12+34,12,34) \in X$. Then the isotropy subgroup of $f_{1}$ is the dihedral group $D_{4}=\langle(1,3,2,4),(1,2)\rangle$ and the orbit of $f_{1}$ is $X_{1}=\left\{f_{1}, f_{2}=p(13+24,13,24), f_{3}=p(14+23,14,23)\right\}$. Moreover, the cyclic group $\langle(2,3,4)\rangle$ acts transitively on $X_{1}$.

Remark Note that the isotropy subgroup of $f_{2}=p(13+24,13,24)$ is $D_{4}^{1}=\langle(1,2,3,4),(1,3)\rangle$ and the isotropy subgroup of $f_{3}=p(14+23,14,23)$ is $D_{4}^{2}=\langle(1,2,4,3),(1,4)\rangle$.

Now we need to transform the $q$-analogue in the definition of $f_{1}=p(12+34,12,34)$ to express it in the form of Euler polynomials.

$$
\begin{aligned}
& {\left[\left(w_{1} w_{2}+w_{3} w_{4}\right) x+w_{1} w_{2} y+w_{3} w_{4} i\right]_{q}} \\
& \quad=\frac{1-q^{\left(w_{1} w_{2}+w_{3} w_{4}\right) x+w_{1} w_{2} y+w_{3} w_{4} i}}{1-q} \\
& =\left(\frac{1-q^{w_{1} w_{2}}}{1-q}\right)\left(\frac{1-q^{\left(w_{1} w_{2}+w_{3} w_{4}\right) x+w_{1} w_{2} y+w_{3} w_{4} i}}{1-q^{w_{1} w_{2}}}\right) \\
& =\left(\frac{1-q^{w_{1} w_{2}}}{1-q}\right)\left(\frac{1-q^{w_{1} w_{2}\left\{\left(1+\frac{w_{3} w_{4}}{w_{1} w_{2}}\right) x+y+\frac{w_{3} w_{4}}{w_{1} w_{2}} i\right\}}}{1-q^{w_{1} w_{2}}}\right) \\
& =\left[w_{1} w_{2}\right]_{q}\left[\left(1+\frac{w_{3} w_{4}}{w_{1} w_{2}}\right) x+y+\frac{w_{3} w_{4}}{w_{1} w_{2}} i\right]_{q^{w_{1}} w_{2}} .
\end{aligned}
$$

Hence

$$
\begin{aligned}
f_{1} & =p(12+34,12,34) \\
& =\sum_{i=0}^{w_{1} w_{2}-1}(-1)^{i} \int_{\mathbb{Z}_{p}} e^{\left[\left(w_{1} w_{2}+w_{3} w_{4}\right) x+w_{1} w_{2} y+w_{3} w_{4} i\right]_{q} t} d \mu_{-1}(y) \\
& =\sum_{n=0}^{\infty} \sum_{i=0}^{w_{1} w_{2}-1}(-1)^{i}\left[w_{1} w_{2}\right]_{q}^{n} E_{n, q^{w}} w_{2}\left(\left(1+\frac{w_{3} w_{4}}{w_{1} w_{2}}\right) x+\frac{w_{3} w_{4}}{w_{1} w_{2}} i\right) \frac{t^{n}}{n !} .
\end{aligned}
$$

Theorem 2.2 For $q \in \mathbb{C}_{p}$ with $|q-1|_{p}<p^{-\frac{1}{p-1}}$ and $n \geq 0$, the modified $q$-Euler polynomials

$$
\sum_{i=0}^{w_{1} w_{2}-1}(-1)^{i}\left[w_{1} w_{2}\right]_{q}^{n} E_{n, q^{w} w_{2}}\left(\left(1+\frac{w_{3} w_{4}}{w_{1} w_{2}}\right) x+\frac{w_{3} w_{4}}{w_{1} w_{2}} i\right)
$$

are invariant under any permutation in the dihedral group $D_{4}=\langle(1,3,2,4),(1,2)\rangle$.

\section{Quadratic symmetry of the modified $q$-Euler polynomials with the isotropy group $V_{4}$}

In this section we investigate two orbits each consisting of six generating functions. 
Let $X_{2}$ be the set of six generating functions $g_{1}=p(13+24,12,34), g_{2}=p(14+23,12,34)$, $g_{3}=p(12+34,13,24), g_{4}=p(14+24,13,24), g_{5}=p(12+34,14,23)$ and $g_{6}=p(13+24,14,23)$ as in the notation of (1.8). That is, each $g_{i}$ is a generating function of the form

$$
p(a b+c d, a c, b d)=\sum_{i=0}^{w_{a} w_{d}-1}(-1)^{i} \int_{\mathbb{Z}_{p}} e^{\left[\left(w_{a} w_{b}+w_{c} w_{d}\right) x+w_{a} w_{c} y+w_{b} w_{d} i\right] q} d \mu_{-1}(y),
$$

where $1 \leq a, b, c, d \leq 4$ and $a, b, c, d$ are distinct.

Note that the action of $S_{3}=\langle(1,2),(1,2,3)\rangle$ on $X_{2}=\left\{g_{1}, \ldots, g_{6}\right\}$ is well defined.

$$
\begin{aligned}
& (1,2)\left(g_{1}\right)=(1,2)(p(13+24,12,34))=p(23+14,21,34)=g_{2}, \\
& (1,3)\left(g_{1}\right)=(1,3)(p(13+24,12,34))=p(31+24,32,14)=g_{6}, \\
& (2,3)\left(g_{1}\right)=(2,3)(p(13+24,12,34))=p(12+34,13,24)=g_{3}, \\
& (1,2,3)\left(g_{1}\right)=(1,2,3)(p(13+24,12,34))=p(21+34,23,14)=g_{5}, \\
& (1,3,2)\left(g_{1}\right)=(1,3,2)(p(13+24,12,34))=p(32+14,31,24)=g_{4} .
\end{aligned}
$$

Consider the normal subgroup $V_{4}=\{(1), \gamma=(1,2)(3,4), \delta=(1,3)(2,4), \gamma \delta=(1,4)(2,3)\}$ of $S_{4}$.

$$
\begin{aligned}
& \gamma\left(g_{1}\right)=(1,2)(3,4)(p(13+24,12,34))=p(24+13,21,34)=g_{1}, \\
& \delta\left(g_{1}\right)=(1,3)(2,4)(p(13+24,12,34))=p(31+42,34,12)=g_{1} .
\end{aligned}
$$

Hence $V_{4}$ fixes $g_{1}$. Since $\left|S_{3}\right| \times\left|V_{4}\right|=\left|S_{4}\right|$, we obtain the following theorem.

Theorem 3.1 Let $X$ be the set of all generating functions of the form

$$
p(a b+c d, j k, \operatorname{lm})=\sum_{i=0}^{w_{j} w_{k}-1}(-1)^{i} \int_{\mathbb{Z}_{p}} e^{\left[\left(w_{a} w_{b}+w_{c} w_{d}\right) x+w_{j} w_{k} y+w_{l} w_{m} i\right]_{q} t} d \mu_{-1}(y)
$$

and $S_{4}$ act on $X$ by permuting $w_{1}, w_{2}, w_{3}, w_{4}$. Take $g_{1}=p(13+24,12,34) \in X$. Then the orbit of $g_{1}$ is $X_{2}=\left\{g_{1}, \ldots, g_{6}\right\}$ and the isotropy subgroup of $g_{1}$ is $V_{4}=\{(1),(1,2)(3,4),(1,3)(2,4)$, $(1,4)(2,3)\}$. Moreover, $S_{3}$ acts transitively on $X_{2}$.

Remark The same computation in (3.3) shows that the isotropy group of each $g_{i}$ is the normal subgroup $V_{4}=\{(1),(1,2)(3,4),(1,3)(2,4),(1,4)(2,3)\}$ of $S_{4}$.

Next we adapt the computation in (2.4) for the change of the $q$-analogue in $g_{1}=$ $p(13+24,12,34)$ and obtain the corresponding modified $q$-Euler polynomials.

$$
\left[\left(w_{1} w_{3}+w_{2} w_{4}\right) x+w_{1} w_{2} y+w_{3} w_{4} i\right]_{q}=\left[w_{1} w_{2}\right]_{q}\left[\left(\frac{w_{3}}{w_{2}}+\frac{w_{4}}{w_{1}}\right) x+y+\frac{w_{3} w_{4}}{w_{1} w_{2}} i\right]_{q^{w_{1}} w_{2}},
$$




$$
\begin{aligned}
g_{1} & =p(13+24,12,34) \\
& =\sum_{i=0}^{w_{1} w_{2}-1}(-1)^{i} \int_{\mathbb{Z}_{p}} e^{\left[\left(w_{1} w_{3}+w_{2} w_{4}\right) x+w_{1} w_{2} y+w_{3} w_{4}\right]_{q} t} d \mu_{-1}(y) \\
& =\sum_{n=0}^{\infty} \sum_{i=0}^{w_{1} w_{2}-1}(-1)^{i}\left[w_{1} w_{2}\right]_{q}^{n} E_{n, q^{w_{1} w_{2}}}\left(\left(\frac{w_{3}}{w_{2}}+\frac{w_{4}}{w_{1}}\right) x+\frac{w_{3} w_{4}}{w_{1} w_{2}} i\right) \frac{t^{n}}{n !} .
\end{aligned}
$$

Theorem 3.2 For $q \in \mathbb{C}_{p}$ with $|q-1|_{p}<p^{-\frac{1}{p-1}}$ and $n \geq 0$, the modified $q$-Euler polynomials

$$
\sum_{i=0}^{w_{1} w_{2}-1}(-1)^{i}\left[w_{1} w_{2}\right]_{q}^{n} E_{n, q^{w_{1}} w_{2}}\left(\left(\frac{w_{3}}{w_{2}}+\frac{w_{4}}{w_{1}}\right) x+\frac{w_{3} w_{4}}{w_{1} w_{2}} i\right)
$$

are invariant under any permutation in the normal subgroup $V_{4}=\{(1),(1,2)(3,4)$, $(1,3)(2,4),(1,4)(2,3)\}$ of $S_{4}$.

Now we consider the set $X_{3}$ of six polynomials $h_{1}=p(12+34,12,12), h_{2}=$ $p(13+24,13,13), h_{3}=p(14+23,14,14), h_{4}=p(12+34,34,34), h_{5}=p(13+24,24,24)$ and $h_{6}=p(14+23,23,23)$. That is, each $h_{i}$ is a polynomial of the form

$$
p(a b+c d, a b, a b)=\sum_{i=0}^{w_{a} w_{b}-1}(-1)^{i} \int_{\mathbb{Z}_{p}} e^{\left[\left(w_{a} w_{b}+w_{c} w_{d}\right) x+w_{a} w_{b} y+w_{a} w_{b} i\right]_{q} t} d \mu_{-1}(y),
$$

where $1 \leq a, b, c, d \leq 4$ and $a, b, c, d$ are distinct.

Now we have the action of $S_{4}$ to collect all of $h_{i}$ in an orbit.

$$
\begin{aligned}
& (2,3)\left(h_{1}\right)=(2,3)(p(12+34,12,12))=p(13+24,13,13)=h_{2}, \\
& (2,4)\left(h_{1}\right)=(2,3)(p(12+34,12,12))=p(14+32,14,14)=h_{3}, \\
& (1,3)(2,4)\left(h_{1}\right)=(1,3)(2,4)(p(12+34,12,12))=(p(34+12,12))=h_{4}, \\
& (1,2,4)\left(h_{1}\right)=(1,2,4)(p(12+34,12,12))=(p(24+31,24,24))=h_{5}, \\
& (1,2,3)\left(h_{1}\right)=(1,2,3)(p(12+34,12,12))=(p(23+14,23,23))=h_{6} .
\end{aligned}
$$

It is obvious that each permutation in the subgroup $\widehat{V}_{4}=\langle(1,2),(3,4)\rangle$ of $S_{4}$ fixes $h_{1}$. As $\left|\hat{V}_{4}\right| \times 6=24$, we conclude the following.

Theorem 3.3 Let $X$ be the set of all generating functions of the form

$$
p(a b+c d, j k, l m)=\sum_{i=0}^{w_{j} w_{k}-1}(-1)^{i} \int_{\mathbb{Z}_{p}} e^{\left[\left(w_{a} w_{b}+w_{c} w_{d}\right) x+w_{j} w_{k} y+w_{l} w_{m} i\right]_{q} t} d \mu_{-1}(y)
$$

and $S_{4}$ act on $X$ by permuting $w_{1}, w_{2}, w_{3}, w_{4}$. Take $h_{1}=p(12+34,12,12) \in X$. Then the orbit of $h_{1}$ is $X_{3}=\left\{h_{1}, \ldots, h_{6}\right\}$ and the isotropy subgroup of $h_{1}$ is $\widehat{V}_{4}=\langle(1,2),(3,4)\rangle$ in $S_{4}$.

Remark The subgroup $\widehat{V}_{4}=\langle(1,2),(3,4)\rangle$ is not normal in $S_{4}$. Further, none of four subgroups of order 6 in $S_{4}$ act transitively on $X_{3}$. 
A slight change of the formula in (2.4) makes $h_{1}=p(13+24,12,34)$ to be modified $q$ Euler polynomials.

$$
\begin{aligned}
& {\left[\left(w_{1} w_{2}+w_{3} w_{4}\right) x+w_{1} w_{2} y+w_{1} w_{2} i\right]_{q}=\left[w_{1} w_{2}\right]_{q}\left[\left(1+\frac{w_{3} w_{4}}{w_{1} w_{2}}\right) x+y+i\right]_{q^{w_{1} w_{2}}},} \\
& h_{1}=p(12+34,12,12) \\
& =\sum_{i=0}^{w_{1} w_{2}-1}(-1)^{i} \int_{\mathbb{Z}_{p}} e^{\left[\left(w_{1} w_{2}+w_{3} w_{4}\right) x+w_{1} w_{2} y+w_{1} w_{2} i\right]_{q} t} d \mu_{-1}(y) \\
& =\sum_{n=0}^{\infty} \sum_{i=0}^{w_{1} w_{2}-1}(-1)^{i}\left[w_{1} w_{2}\right]_{q}^{n} E_{n, q^{w_{1} w_{2}}}\left(\left(1+\frac{w_{3} w_{4}}{w_{1} w_{2}}\right) x+i\right) \frac{t^{n}}{n !}
\end{aligned}
$$

Theorem 3.4 For $q \in \mathbb{C}_{p}$ with $|q-1|_{p}<p^{-\frac{1}{p-1}}$ and $n \geq 0$, the modified $q$-Euler polynomials

$$
\sum_{i=0}^{w_{1} w_{2}-1}(-1)^{i}\left[w_{1} w_{2}\right]_{q}^{n} E_{n, q^{w_{1} w_{2}}}\left(\left(1+\frac{w_{3} w_{4}}{w_{1} w_{2}}\right) x+i\right)
$$

are invariant under any permutation in the subgroup $\widehat{V}_{4}=\langle(1,2),(3,4)\rangle$ of $S_{4}$.

\section{Less symmetric modified $q$-Euler polynomials}

When $S_{4}$ acts on the set $X$ of 315 generating functions listed in (1.8), there are seven orbits having 12 generating functions and nine orbits having 24 generating functions. That is, these are the generating functions whose isotropy subgroup is a cyclic group of order 2 or a trivial group. In this section we just illustrate a typical one for each case.

Let $X_{4}$ be the set of all generating functions of the form

$$
p(a c+a d, a b, a b)=\sum_{i=0}^{w_{a} w_{b}-1}(-1)^{i} \int_{\mathbb{Z}_{p}} e^{\left[\left(w_{a} w_{c}+w_{a} w_{d}\right) x+w_{a} w_{b} y+w_{a} w_{b} i\right]_{q} t} d \mu_{-1}(y),
$$

where $1 \leq a, b, c, d \leq 4$ and $a, b, c, d$ are distinct.

That is, $X_{4}$ consists of the following 12 polynomials:

$$
\begin{array}{ll}
f_{1}=p(13+14,12,12), & f_{2}=p(23+24,12,12), \\
f_{3}=p(12+14,13,13), & f_{4}=p(32+34,13,13), \\
f_{5}=p(12+13,14,14), & f_{6}=p(42+43,14,14), \\
f_{7}=p(21+24,23,23), & f_{8}=p(31+34,23,23), \\
f_{9}=p(21+23,24,24), & f_{10}=p(41+43,24,24), \\
f_{11}=p(31+32,34,34), & f_{12}=p(41+42,34,34) .
\end{array}
$$


We can check that the alternating group $A_{4}$ acts on $X_{4}$ transitively.

$$
\begin{array}{ll}
(1,2,3) f_{1}=f_{7}, & (1,3,2) f_{1}=f_{4}, \\
(1,2,4) f_{1}=f_{9}, & (1,4,2) f_{1}=f_{6}, \\
(1,3,4) f_{1}=f_{8}, & (1,4,3) f_{1}=f_{10}, \\
(2,3,4) f_{1}=f_{3}, & (2,4,3) f_{1}=f_{5}, \\
(1,2)(3,4) f_{1}=f_{2}, & (1,3)(2,4)) f_{1}=f_{11}, \\
(1,4)(2,3)) f_{1}=f_{12} . &
\end{array}
$$

As $(3,4) f_{1}=(3,4)(p(13+14,12,12))=p(14+13,12,12)=f_{1}$, the isotropy subgroup of $f_{1}$ is $\langle(3,4)\rangle$ and $X_{4}$ is the orbit of $f$. Note that $\langle(3,4)\rangle$ consists of one even permutation (1) and one odd permutation $(3,4)$. So, for any $p(a c+a d, a b, a b) \in X_{4}$, there is an even permutation $\tau$ such that $\tau\left(f_{1}\right)=p(a c+a d, a b, a b)$. Hence the alternating subgroup $A_{4}$ of $S_{4}$ acts transitively on $X_{4}$.

Theorem 4.1 Let $X$ be the set of all generating functions of the form

$$
p(a b+c d, j k, \operatorname{lm})=\sum_{i=0}^{w_{j} w_{k}-1}(-1)^{i} \int_{\mathbb{Z}_{p}} e^{\left[\left(w_{a} w_{b}+w_{c} w_{d}\right) x+w_{j} w_{k} y+w_{l} w_{m} i\right]_{q} t} d \mu_{-1}(y)
$$

and $S_{4}$ act on $X$ by permuting $w_{1}, w_{2}, w_{3}, w_{4}$. Take $f_{1}=p(13+14,12,12) \in X$. Then the orbit of $f_{1}$ is $X_{4}$ consisting of 12 polynomials as above and the isotropy subgroup of $f_{1}$ is $\langle(3,4)\rangle$. Moreover, $A_{4}$ acts transitively on $X_{4}$.

Finally, we illustrate one of the nine orbits each consisting of 24 generating functions. Let $X_{5}$ be the set of all generating functions of the form

$$
p(a b+a c, a b, a b)=\sum_{i=0}^{w_{a} w_{b}-1}(-1)^{i} \int_{\mathbb{Z}_{p}} e^{\left[\left(w_{a} w_{b}+w_{a} w_{c}\right) x+w_{a} w_{b} y+w_{a} w_{b} i\right]_{q} t} d \mu_{-1}(y),
$$

where $1 \leq a, b, c \leq 4$ and $a, b, c$ are distinct. Note that there are six choices for the coefficient $w_{a} w_{b}$ of $y$ and four choices for the coefficient $w_{a} w_{b}+w_{a} w_{c}$ of $x$. Hence $X_{5}$ consists of 24 generating functions. We use $g=p(12+13,12,12) \in X_{5}$, then we can see that the action of $S_{4}$ on $X_{5}$ is transitive.

\section{Conclusion}

In this paper we investigate the symmetric property of the Euler polynomials. If we use $n$ weights $w_{1}, \ldots, w_{n}$ in the definition of the generating functions of Euler polynomials, then the symmetric group $S_{n}$ naturally acts on a prescribed set of generating functions of the Euler polynomials. This paper uses four weights $w_{1}, \ldots, w_{4}$ in a quadratic form. We find that the dihedral group $D_{4}$ (or the Klein 4 group $V_{4}$ ) is an isotropy group of some generating function. As a result, the corresponding Euler polynomial is fixed by $D_{4}$ (or $\left.V_{4}\right)$. The results in the paper extend to other special polynomials such as degenerate Euler polynomials and Catalan polynomials. It is a further problem to find special polynomials that are invariant under the alternating group $A_{n}$. 


\section{Acknowledgements}

This work was supported by the National Research Foundation of Korea (NRF) grant funded by the Korea government (MEST) (No. 2017R1E1A1A03070882).

\section{Competing interests}

The authors declare that they have no competing interests.

\section{Authors' contributions}

All authors contributed equally to the manuscript and typed, read and approved the final manuscript.

\section{Author details}

'Department of Mathematics Education, Konkuk University, Seoul, Republic of Korea. ${ }^{2}$ Department of Mathematics, Kwangwoon University, Seoul, Republic of Korea. ${ }^{3}$ Department of Mathematics Education and ERl, Gyeongsang National University, Jinju, Republic of Korea.

\section{Publisher's Note}

Springer Nature remains neutral with regard to jurisdictional claims in published maps and institutional affiliations.

Received: 20 November 2017 Accepted: 15 January 2018 Published online: 24 January 2018

\section{References}

1. Carlitz, L: q-Bernoulli and Eulerian numbers. Trans. Am. Math. Soc. 76, 332-350 (1954)

2. Kim, T: q-Volkenborn integration. Russ. J. Math. Phys. 9(3), 288-299 (2002)

3. Babaei, E, Zamani, Y: Symmetry classes of polynomials associated with the dihedral group. Bull. Iran. Math. Soc. 40(4), 863-874 (2014)

4. Cohen, H: Number Theory, Vol. II: Analytic and Modern Tools. Graduate Texts in Mathematics, vol. 240. Springer, New York (2007)

5. Fraleigh, JB: A First Course in Abstract Algebra. Pearson, Upper Saddle River (2003)

6. Herstein, IN: Topics in Algebra. Wiley, New York (2006)

7. Kim, DS, Kim, T: Identities of symmetry for generalized $q$-Euler polynomials arising from multivariate fermionic $p$-adic integral on $\mathbb{Z}_{p}$. Proc. Jangjeon Math. Soc. 17(4), 519-525 (2014)

8. Kim, DS, Lee, $\mathrm{N}, \mathrm{Na}$, J, Park, KH: Identities of symmetry for higher-order Euler polynomials in three variables (I). Adv. Stud. Contemp. Math. (Kyungshang) 22(1), 51-74 (2012)

9. Kim, DS, Lee, N, Na, J, Park, KH: Abundant symmetry for higher-order Bernoulli polynomials (I). Adv. Stud. Contemp. Math. (Kyungshang) 23(3), 461-482 (2013)

10. Kim, DS: Symmetry identities for generalized twisted Euler polynomials twisted by unramified roots of unity. Proc Jangjeon Math. Soc. 15(3), 303-316 (2012)

11. Kim, T: Symmetry $p$-adic invariant integral on $\mathbb{Z}_{p}$ for Bernoulli and Euler polynomials. J. Differ. Equ. Appl. 14(12), 1267-1277 (2008)

12. Kim, T: Symmetry of power sum polynomials and multivariate fermionic $p$-adic invariant integral on $\mathbb{Z}_{p}$. Russ. J. Math. Phys. 16(1), 93-96 (2009)

13. Kim, T, Dolgy, DV, Jang, L-C, Kwon, HI: Some identities of $q$-Euler polynomials under the symmetry group of degree $n$. J. Nonlinear Sci. Appl. 9, 1077-1082 (2016)

14. Kim, T, Hwang, K-W, Kim, Y-H: Symmetry properties of higher-order Bernoulli polynomials. Adv. Differ. Equ. 2009 Article ID 318639 (2009)

15. Pinter, CC: A Book of Abstract Algebra. Dover, Mineola (2012)

16. Schikhof, WH: Ultrametric Calculus: An Introduction to $p$-Adic Analysis. Cambridge University Press, Cambridge (2006)

17. Tuenter, HJH: A symmetry of power sum polynomials and Bernoulli numbers. Am. Math. Mon. 108(3), $258-261$ (2001)

18. He, Y: Symmetric identities for Carlitz's q-Bernoulli numbers and polynomials. Adv. Differ. Equ. 2013, Article ID 246 (2013)

19. /Simsek, Y: Complete sum of products of $(h, q)$-extension of Euler polynomials and numbers. J. Differ. Equ. Appl. 16(11), 1331-1348 (2010)

20. Kim, T, Kim, DS: Identities of symmetry for degenerate Euler polynomials and alternating generalized falling factorial sums. Iranian Sci. Tech. Trans. A-Sci. 41, 939-949 (2017) 